\title{
Non-small-cell Lung Cancer with Severe Skin Manifestations Related to Radiation Recall Dermatitis after Atezolizumab Treatment
}

\author{
Kei Nakashima ${ }^{1}$, Koichi Saruwatari ${ }^{1}$, Ryo Sato ${ }^{1}$, Kosuke Imamura ${ }^{1}$, Ikko Kajihara ${ }^{2}$, \\ Satoshi Fukushima ${ }^{2}$, Tetsuo Saito ${ }^{3}$, Shiho Ishizuka ${ }^{1}$, Daisuke Tamanoi ${ }^{1}$, Takayuki Jodai ${ }^{1}$, \\ Shohei Hamada ${ }^{1}$, Yusuke Tomita ${ }^{1}$, Sho Saeki ${ }^{1}$, Hidenori Ichiyasu ${ }^{1}$, Natsuo Oya ${ }^{3}$, \\ Hironobu $\mathrm{Ihn}^{2}$ and Takuro Sakagami ${ }^{1}$
}

\begin{abstract}
:
Radiation recall dermatitis (RRD) is an inflammatory reaction that occurs at previously irradiated skin regions after drug administration. We herein report a patient with non-small-cell lung cancer treated previously with thoracic radiotherapy who developed severe RRD induced by atezolizumab [anti-programmed death 1 ligand 1 (PD-L1) antibody]. Immunohistochemistry of the skin biopsy showed dermatitis with infiltration of CD8+ lymphocytes, suggesting that atezolizumab might provoke an immune-related inflammatory reaction at previously irradiated skin regions. When administering anti-PD-L1 antibody to patients who have undergone radiotherapy previously, physicians should carefully monitor the irradiated skin for the potential occurrence of RRD.
\end{abstract}

Key words: radiation recall dermatitis, non-small cell lung cancer, atezolizumab

(Intern Med 59: 1199-1202, 2020)

(DOI: 10.2169/internalmedicine.3937-19)

\section{Introduction}

Immune checkpoint inhibitors, including programmed cell death-1/programmed cell death ligand-1 (PD-1/PD-L1), provide dramatic survival benefits to patients with advanced non-small-cell lung cancer (NSCLC). Atezolizumab, a humanized, engineered immunoglobulin-G1 monoclonal antibody targeting PD-L1, has demonstrated improved survival rates over docetaxel in previously treated patients with NSCLC (1). However, immune checkpoint inhibitors like atezolizumab lead to various immune-related adverse events (irAEs) by increasing the activity of the immune system (2). Immune checkpoint inhibitor-induced skin toxicities are the most frequent irAE, and inflammatory dermatosis conditions, including erythema multiforme minor, lichenoid, and eczematous dermatitis, occasionally cause life-threatening events, such as Stevens-Johnson syndrome (3-5).

Radiation recall dermatitis (RRD) is an acute inflammatory skin reaction in a previously irradiated area triggered by the administration of a systemic agent. RRD is a relatively rare phenomenon and develops within days to weeks after drug administration $(6,7)$. Although most recall-triggering drugs are cytotoxic anticancer agents, information on RRD induced by immune checkpoint inhibitors is scanty.

We herein report a patient with NSCLC who developed severe skin manifestations related to RRD after the administration of atezolizumab. To evaluate the immune microenvironment of RRD, we performed an immunohistochemistry analysis of a skin biopsy based on T lymphocytes and the PD-L1 expression.

${ }^{1}$ Department of Respiratory Medicine, Kumamoto University Hospital, Japan, ${ }^{2}$ Department of Dermatology and Plastic Surgery, Kumamoto University Hospital, Japan and ${ }^{3}$ Department of Radiation Oncology, Kumamoto University Hospital, Japan

Received: September 17, 2019; Accepted: December 12, 2019; Advance Publication by J-STAGE: February 12, 2020

Correspondence to Dr. Koichi Saruwatari, ksaruwat@kuh.kumamoto-u.ac.jp 
(A)

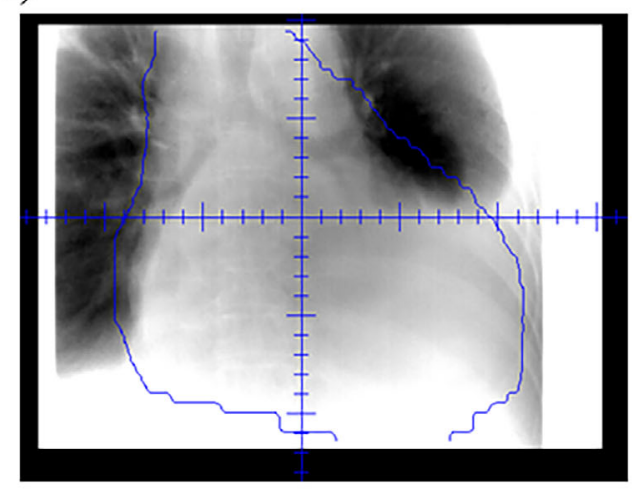

(B)

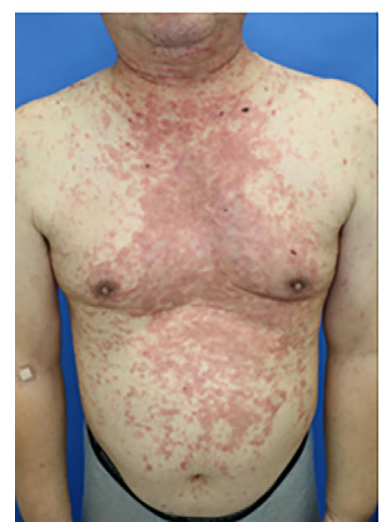

(C)

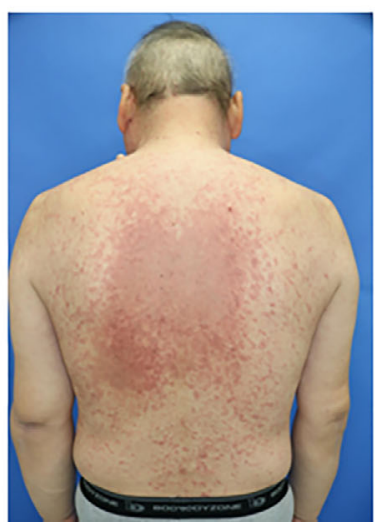

(D)

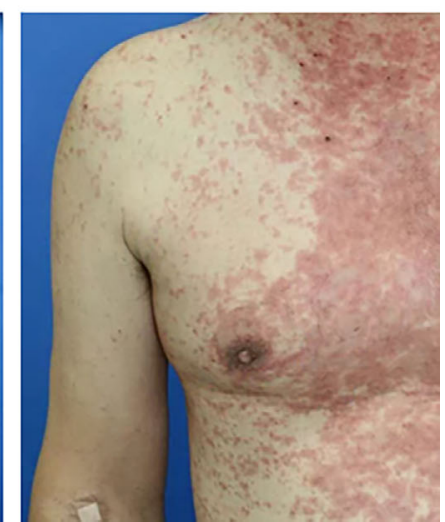

Figure 1. Clinical features of radiation recall dermatitis after atezolizumab treatment. (A) Portal radiographs of radiotherapy for enlarged lymph nodes and pericardium. (B) (C) Relatively well-circumscribed erythema at the site of previous irradiation over the chest and abdominal regions. (D) Detailed view of a rash with red and raised patches.

\section{Case Report}

A 61-year-old man was diagnosed with clinical T3N2M0 stage IIIC lung squamous cell carcinoma with a PD-L1 expression of $1 \%$ in June 2018. Chemotherapy with carboplatin and nab-paclitaxel was administered as first-line treatment. After the initial treatment, however, he developed difficulty breathing with symptoms of superior vena cava syndrome caused by enlarged mediastinal lymph nodes and carcinomatous pericarditis. He received $1 \mathrm{mg}$ of oral dexamethasone and palliative radiotherapy for lymph node metastases and pericardium with a total dose of $30 \mathrm{~Gy}$ in daily fractions of 3 Gy (Fig. 1A).

Although the symptoms of breathlessness improved after radiotherapy, a computed tomography scan showed progression of adrenal metastasis. Subsequently, he was treated with intravenous infusion of 1,200 $\mathrm{mg}$ atezolizumab. Twenty-one days after the administration of atezolizumab, he was admitted with the occurrence of relatively well-circumscribed erythema at the previously irradiated skin field (Fig. 1B). An erythematous rash was mainly observed in the trunk, part of the upper extremities, and the face and not in the lower extremities, nails, and oral cavity. Signs of infection, autoim- mune disorders, and suspected use of agents aside from antiPD-L1 antibody were absent. A skin biopsy showed interface dermatitis with perivascular lymphocytic inflammatory cell infiltration (Fig. 2A). Based on these findings, we diagnosed him with severe skin disorder (CTCAE version 5.0, Grade 3) related to RRD induced by atezolizumab treatment.

He discontinued atezolizumab treatment and received an intravenous 150-mg dose of methylprednisolone (2 mg/kg/ day) for 3 days. Subsequently, he was treated with an intravenous $75 \mathrm{mg}$ dose of methylprednisolone $(1 \mathrm{mg} / \mathrm{kg} / \mathrm{day})$ for 4 days, and then oral prednisolone was reduced to $40 \mathrm{mg}$ / day. Prednisolone was tapered by $10 \mathrm{mg}$ per week and stopped 4 weeks after the occurrence of RRD. His skin disorders disappeared two weeks after steroid therapy initiation and did not relapse.

\section{Discussion}

To the best of our knowledge, this is the first reported case of NSCLC in which severe skin manifestations associated with RRD occurred after administration of atezolizumab.

As mentioned above, RRD is an inflammatory skin disease that occurs in a previously irradiated field following the 

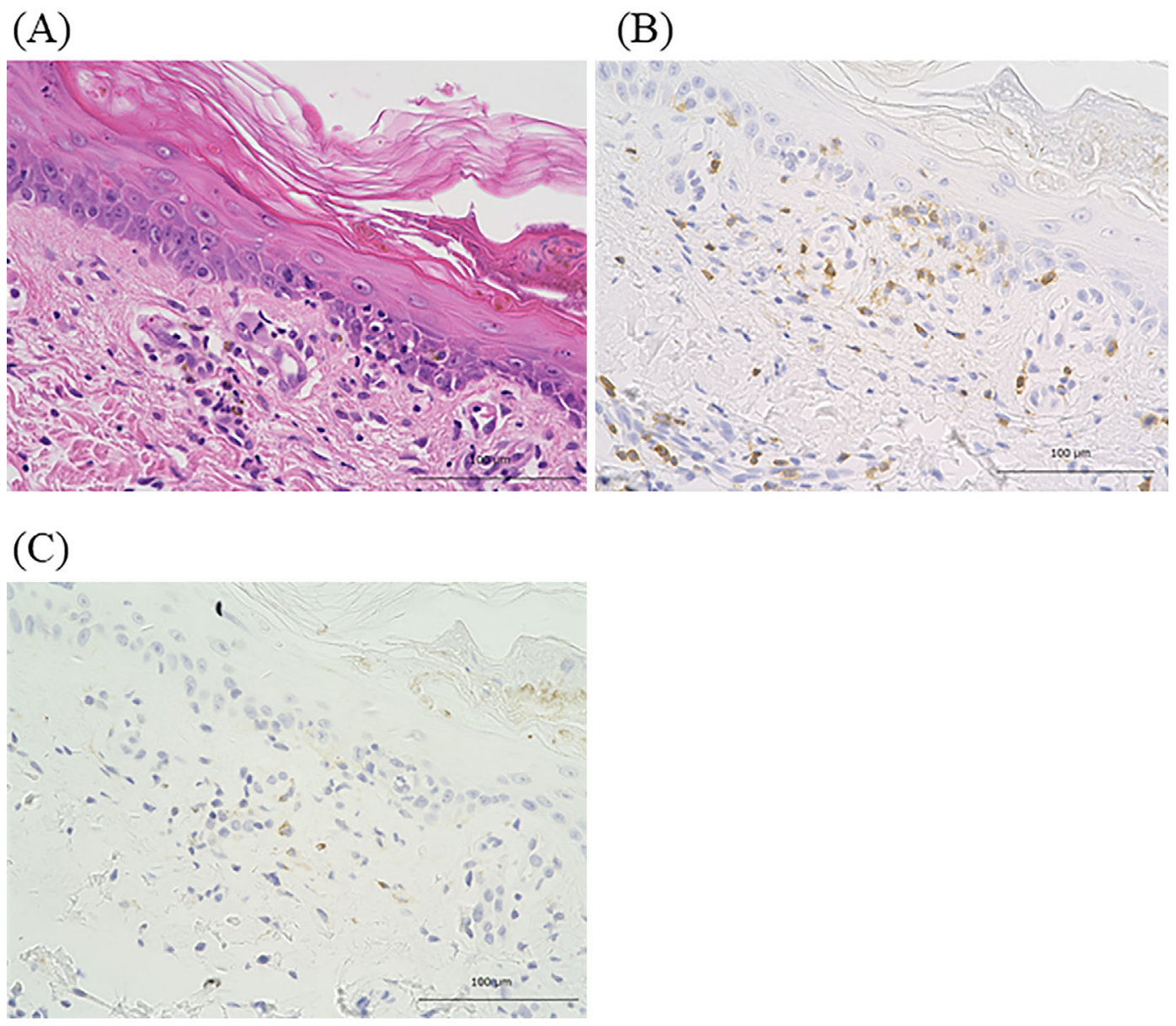

Figure 2. Results of the histologic analysis of a skin biopsy sample. Hematoxylin and Eosin staining (A) (original magnification $\times 400$ ). Immunohistochemical staining for CD8 [Clone C8/144B (Nichirei Bioscience, Tokyo, Japan)] (B) (original magnification $\times 400$ ) and PD-L1 [Clone E1 L3N (Cell Signaling Technology, Danvers, USA)] (C) (original magnification $\times 400)$.

administration of systemic agents, such as cytotoxic chemotherapeutic agents. RRD occurrence can be considered similar to that of the Koebner phenomenon, which involves the appearance of skin lesions at the site of injuries, such as drug reactions and irradiation $(6,8)$. The severity of RRD is mostly mild or moderate but can occasionally be severe. The interval between drug administration and the onset of RRD is several days to weeks $(6,7)$. Recently, in three reports of RRD induced by nivolumab (anti-PD-1 antibody), two out of three patients developed life-threating dermatitis with Stevens-Johnson syndrome, and the interval from nivolumab administration to the onset of RRD was between three days and two weeks (9-11). In the present case, the grade of RRD was severe, and the interval between atezolizumab administration and the onset of RRD was three weeks, which is consistent with these previous nivolumab-induced RRD case reports. In our case, the skin disorders improved after the administration of high-dose corticosteroids, despite the severe skin toxicity of the irAE. Although the role of systematic steroid therapy in RRD is unclear, management in line with the guidelines of immune-related skin disorders (prednisolone 1-2 mg/kg, tapering over at least 4 weeks) is preferred for severe RRD induced by immune checkpoint in- hibitors $(3,4)$. Recently, PACIFIC study showed that durvalamb, an anti-PD-L1 antibody, improved the overall survival in patients with locally advanced NSCLC after chemoradiotherapy (12). Opportunities for the administration of anti-PD-L1 antibody have therefore been increasing in patients with NSCLC who received thoracic radiotherapy. When administering immune checkpoint inhibitors in patients previously treated with radiotherapy, skin reactions at the irradiated fields should be monitored closely, especially in the early periods after these treatments.

The precise mechanism underlying RRD induced by antiPD-L1 antibody is unknown. However, for irAEs associated with immune checkpoint inhibitors, some potential mechanisms have been suggested, including increased T-cell activity against antigens presenting in tumors and healthy tissue, increased levels of preexisting autoantibodies or inflammatory cytokines, and enhanced complement-mediated inflammation due to direct binding of immune checkpoint inhibitors with immune checkpoint molecules expressed on normal tissue (2). With respect to immune-related skin reactions, Goldinger et al. analyzed the immunohistochemistry of skin biopsy samples from melanoma patients who developed cutaneous adverse events due to anti-PD-1 antibody 
and found that CD8-positive lymphocytes had accumulated at the dermal junction and keratinocytes were expressed with PD-L1 (13). In the present case, the immunohistochemical analysis showed that cytotoxic CD8-positive lymphocytes had infiltrated the epidermal-dermal junction and perivascular lesions, although the PD-L1 expression on epidermal cells was not detected (Fig. 2B, C). Blocking the interaction between PD-1 and PD-L1 is involved in the effector phase of the immune response as well as the priming phase of naïve CD8-positive $\mathrm{T}$ cells because of the presence of PD-L1 expression on the surface of dendric cells in the lymph nodes (14). Based on these findings, we hypothesize that RRD was induced by anti-PD-L1 antibody by activated cytotoxic lymphocytes recognizing the irradiated skin epithelium as non-self antigens in the priming phase and then infiltrating these regions to induce an immune-related inflammatory response.

\section{Conclusion}

We encountered a patient with NSCLC who developed severe skin reactions related to RRD after atezolizumab administration. We detected the infiltration of cytotoxic lymphocytes in a skin biopsy sample via an immunohistochemical analysis. When administering anti-PD-L1 antibody to patients with a history of radiotherapy, physicians should carefully check for skin reactions at the irradiated field, keeping in mind the possibility of RRD.

The authors state that they have no Conflict of Interest (COI).

\section{Acknowledgement}

We are grateful to Ms. Misako Takahashi, who is a research assistant in the Department of Respiratory Medicine at $\mathrm{Ku}-$ mamoto University Hospital, for her support.

\section{References}

1. Facchinetti F, Bordi P, Leonetti A, Buti S, Tiseo M. Profile of atezolizumab in the treatment of metastatic non-small-cell lung cancer: patient selection and perspectives. Drug Des Devel Ther 12: 2857-2873, 2018.
2. Postow MA, Sidlow R, Hellmann MD. Immune-related adverse events associated with immune checkpoint blockade. New Engl J Med 378: 158-168, 2018.

3. Haanen J, Carbonnel F, Robert C, et al. Management of toxicities from immunotherapy: ESMO Clinical Practice Guidelines for diagnosis, treatment and follow-up. Ann Oncol 28 (Suppl 4): iv119iv142, 2017.

4. Brahmer JR, Lacchetti C, Schneider BJ, et al. Management of immune-related adverse events in patients treated with immune checkpoint inhibitor therapy: American Society of Clinical Oncology Clinical Practice Guideline. J clinical oncology: official journal of the American Society of Clinical Oncology 36: 1714-1768, 2018.

5. Lacouture M, Sibaud V. Toxic side effects of targeted therapies and immunotherapies affecting the skin, oral mucosa, hair, and nails. Am J Clin Dermatol 19 (Suppl 1): 31-39, 2018.

6. Camidge R, Price A. Characterizing the phenomenon of radiation recall dermatitis. Radiother Oncol 59: 237-245, 2001.

7. Burris HA 3rd, Hurtig J. Radiation recall with anticancer agents. Oncologist 15: 1227-1237, 2010.

8. Sagi L, Trau H. The Koebner phenomenon. Clin Dermatol 29: 231-236, 2011.

9. Korman AM, Tyler KH, Kaffenberger BH. Radiation recall dermatitis associated with nivolumab for metastatic malignant melanoma. Int J Dermatol 56: e75-e77, 2017.

10. Rouyer L, Bursztejn AC, Charbit L, Schmutz JL, Moawad S. Stevens-Johnson syndrome associated with radiation recall dermatitis in a patient treated with nivolumab. Eur J Dermatol 28: 380$381,2018$.

11. Shah KM, Rancour EA, Al-Omari A, Rahnama-Moghadam S. Striking enhancement at the site of radiation for nivolumabinduced Stevens-Johnson syndrome. Dermatol Online J. Forthcoming.

12. Antonia SJ, Villegas A, Daniel D, et al. Overall survival with durvalumab after chemoradiotherapy in stage III NSCLC. New Engl J Med 379: 2342-2350, 2018.

13. Goldinger SM, Stieger P, Meier B, et al. Cytotoxic cutaneous adverse drug reactions during anti-PD-1 therapy. Clin Cancer Res 22: 4023-4029, 2016.

14. Gibbons Johnson RM, Dong H. Functional expression of programmed death-ligand 1 (B7-H1) by immune cells and tumor cells. Front Immunol 8: 961, 2017.

The Internal Medicine is an Open Access journal distributed under the Creative Commons Attribution-NonCommercial-NoDerivatives 4.0 International License. To view the details of this license, please visit (https://creativecommons.org/licenses/ by-nc-nd/4.0/). 